\title{
Reflexiones acerca de la Cooperación Sur-Sur Descentralizada en el caso de las zonas de frontera del Mercosur
}

\section{Reflections on Decentralized South-South Cooperation in the case of the border areas of Mercosur}

ARTÍCULO

\begin{abstract}
Debora Fagaburu
Consejo Nacional de Investigaciones Científicas y Técnicas. Universidad Nacional de Quilmes. Contacto: deborafagaburu@gmail.com
\end{abstract}

Recibido: junio de 2018

Aceptado: julio de 2018

\begin{abstract}
Resumen
La Cooperación Sur-Sur (CSS) Descentralizada es una modalidad de la Cooperación Internacional al Desarrollo que ha venido intensificándose en las últimas décadas. Este trabajo pretende reflexionar de manera crítica sobre esta cooperación en la región de América Latina y adoptar un camino de abordaje para futuras investigaciones en el sub-campo de la agenda educativa regional y transfronteriza, particularmente para el caso del Mercado Común del Sur (Mercosur). En primer lugar, se realiza un mapeo de conceptos vinculados a la temática, que dada su carácter multidimensional, abarca postulados de las Relaciones Internacionales, de los enfoques sobre regionalismo y de gobernanza. Luego, se tratan las especificidades de la CSS Descentralizada en la región latinoamericana y se abordan las características de la misma vinculada a la agenda educativa. Para terminar, este estudio plantea la necesidad de reconocer los desafíos y oportunidades de esta modalidad específica de CSS, la cual desde el territorio puede contribuir a crear oportunidades genuinas para un modelo regional de carácter positivo.
\end{abstract}

Palabras clave: Cooperación Sur-Sur Descentralizada; Mercosur; Educación.

\section{Abstract}

South-South Cooperation (CSS) Decentralized is a modality of International Development Cooperation that has been intensifying in recent decades. This paper intends to reflect critically on this cooperation in the Latin American region and adopt a path for future research in the sub-field of the regional and cross-border educational agenda, particularly in the case of the Common Market of the South (Mercosur). In the first place, a mapping of concepts linked to the subject is carried out, which, given its multidimensional nature, encompasses postulates of International Relations, approaches to regionalism and governance. Then, the specificities of the Decentralized CSS in the Latin American region are discussed and the characteristics of the same linked to the educational agenda are addressed. Finally, this study raises the need to recognize the challenges and opportunities of this specific form of CSS, which from the territory can contribute to create genuine opportunities for a positive regional model.

Keywords: Decentralized South-South Cooperation; Mercosur; Education.

\section{INTRODUCCIÓN}

En tanto política pública, las políticas de Cooperación Internacional $(\mathrm{Cl})$, se pueden abordar como un instrumento para fortalecer y complementar las capacidades nacionales en múltiples 
sectores, con vistas a lograr mejoras en la calidad de vida de la sociedad, e incentivar al mismo tiempo los proyectos en la arena regional. En el marco de la Cooperación Internacional al Desarrollo (CID), las políticas públicas en educación pueden incidir directamente en el bienestar y la inclusión social, dada su tendencia a democratizar y dimensionar desde el territorio los desafíos que presentan problemas sociales como la inclusión educativa (Arias, 2011). En este sentido radica la relevancia de ampliar el abanico de abordajes en el sub-campo de conocimiento y debate vinculado a la Cooperación Sur-Sur (CSS) en educación, a los fines de aportar a perspectivas integrales sobre la planificación del proceso regional desde la dimensión social y local en América Latina. En los últimos años, una gran parte de la producción de conocimiento de la CSS latinoamericana se ha orientado a tomar como objeto de estudio su vínculo con la integración regional y los diversos sectores de la agenda social (Geneiro \& Vázquez, 2006; Arias, 2011; Perrota, 2012; Barragán, 2015).

Sin embargo, la CSS y su modalidad Descentralizada en el área educativa vinculada a los procesos regionales latinoamericanos y su gobernanza, así como a las tensiones sociales, culturales, y políticas producidas por la coordinación regional en cada territorio, son áreas temáticas en las que aún resta explorar. Se considera que este trabajo es una primera aproximación a las problemáticas vinculadas a la agenda de la CSS Descentralizada en educación, en el marco de las fronteras del Mercosur. En este sentido, se espera contribuir en el futuro a líneas de investigación relacionadas a los efectos de la coordinación regional de políticas públicas sobre el diseño, implementación y gestión de las políticas públicas educativas nacionales de los socios del bloque.

En un artículo anterior denominado "Afinidades entre la Cooperación Internacional y la Integración Regional en América Latina" (2017), la autora ha realizado una revisión crítica de la literatura vinculada a la temática en cuestión. Con aquel repaso teórico-conceptual ya desarrollado, el presente escrito busca reflexionar críticamente acerca de la CSS Descentralizada y adoptar un camino de abordaje para futuras investigaciones en el sub-campo de la agenda educativa regional y transfronteriza, particularmente para el caso del Mercado Común del Sur (Mercosur). En el segundo apartado se propone un mapeo de conceptos utilizados en los distintos enfoques para estudiar el sub-campo de conocimiento y debate mencionado. En el tercer apartado, se trata las especificidades de la CSS en su modalidad descentralizada en América Latina y se abordan las caracteristicas de la misma vinculada a la agenda educativa.

Particularmente, dada la coyuntura internacional actual, se acepta que el institucionalismo neoliberal o neoliberalismo es uno de los enfoques teóricos relativamente más adecuado para pensar la cooperación internacional. Este explica, desde las Relaciones Internacionales, que el Estado es el principal actor de la política mundial, actor egoísta y racional cuyo comportamiento es influenciado por la existencia de instituciones supranacionales que garantizan el cumplimiento de los acuerdos celebrados en el marco de un sistema anárquico (Fagaburu, 2017). Es decir, la 
cooperación tiene lugar cuando las políticas públicas desarrolladas por un gobierno son percibidas por sus socios como estimulantes para la realización de sus propios objetivos, como resultado de un proceso de coordinación de políticas (Keohane, 1993). Especialmente, la CSS es abordada en el sub-apartado 2.1. como una coordinación de políticas entre países en desarrollo, en búsqueda de armonizar intereses conflictivos y complementarios. Específicamente, la cooperación descentralizada en zonas de frontera posee motivaciones ligadas al desarrollo territorial, la seguridad, el comercio internacional y la infraestructura de conectividad física (Rhi-Sausi \& Oddone, 2013).

Si bien la cooperación descentralizada se ha implementado bajo una articulación NorteSur, su rol en iniciativas Sur-Sur se viene consolidando en los últimos años de manera acertada y progresiva. El primer país de América Latina que, a fines de los años 60, implementó acuerdos de hermanamiento con ciudades africanas fue Brasil. Ello sentó las bases para la descentralización de la CSS que se desarrolla actualmente (SEGIB, 2016), la cual se entiende como una modalidad de CID y se define de la siguiente manera:

Una cooperación que se caracteriza por la manera de compartir mutuamente conocimientos, habilidades, experiencia y recursos humanos, técnicos, económicos y/o financieros para alcanzar objetivos de desarrollo local o regional, a través de esfuerzos concertados entre los gobiernos y otros actores subnacionales involucrados (SEGIB, 2017, p. 33).

En el caso de las acciones en el sector educativo, la cooperación descentralizada y transfronteriza se suele vincular a las políticas nacionales sobre desarrollo social, estrechamente relacionadas a las desigualdades locales. En el marco de un proyecto regional, la importancia del rol activo de las unidades sub-nacionales radica en la idea de anclar la construcción de institucionalidad regional desde el territorio, a través del aumento de las transacciones, la confianza y la lealtad a la región en cuestión. En otras palabras, la paradiplomacia estimula el progreso de un tipo de regionalismo más igualitario a través del desarrollo de una gobernanza transfronteriza con mayor margen para la participación social (Rhi-Sausi \& Oddone, 2013).

En el sub-apartado 3.1, se trata la CSS Descentralizada en vínculo al Sector Educativo del Mercosur. Para el caso del bloque, la cooperación descentralizada/transfronteriza ha estado ligada en sus inicios a la agenda comercial, es decir, a un regionalismo con una agenda principalmente "negativa". Pero a partir de los años 2000, el bloque prioriza la dimensión social del proyecto regional, y la CSS Regional en educación se posiciona como la herramienta por excelencia para fijar la agenda regional de forma "positiva", concibiendo la coordinación regional de políticas educativas como un medio para subsanar las asimetrías regionales. Esta se implementa vía su Sector Educativo (SEM). En términos generales, los temas de agenda del SEM durante 2006-2015, se relacionaron con el fortalecimiento de las instituciones de gestión educativa, la formación docente continua, la renovación educativa, la vinculación de la educación 
con el mercado laboral y la producción, y la cooperación interuniversitaria en lo referido al intercambio docente.

En los últimos 15 años, la CSS del SEM se trató de una coordinación inter-gubernamental de políticas especialmente técnica, vinculada a seminarios, talleres, movilidad docente, movilidad estudiantil en educación superior, capacitaciones para el fortalecimiento institucional, redes de profesionales para profundizar la formación docente, entre otras. Ello fortaleció de forma progresiva los canales de diálogo e intercambio de información y experiencias a nivel sectorial y local, donde se plasman las mayores desigualdades sociales, estrechamente relacionadas a las asimetrías entre los socios. En este sentido, "las relaciones interpersonales entre las poblaciones fronterizas son ambiguas, a veces contradictorias, y remiten, de cierta manera, las asimetrías sociales y económicas locales y entre los países, que se diagnostican en datos estadísticos" (Desiderá Neto \& Penha, 2017, p. 208). Por ello, en las reflexiones finales se apunta a destacar el abanico de posibilidades que brinda la CSS Regional Descentralizada para potenciar desde el territorio, la autonomía relacional de los socios y avanzar en grados de regionidad a partir de la profundización de la agenda social.

\section{DEBATES SOBRE EL REGIONALISMO}

Plantear los conceptos de región y regionalismo importa a los fines de este trabajo y de futuras investigaciones ya que el Mercosur se auto-define como un proyecto político, un tipo específico de regionalismo, que si bien persigue la integración regional, desde sus inicios asociada al plano económico y comercial, en la práctica se identifica con formas de "cooperación regional inter-estatal", que Hurrell (1995) plantea como aquellas situaciones en las que los gobiernos patrocinan los acuerdos para afrontar problemas de interés compartido.

Desde los postulados de Nye (1968), se entiende a la región como un grupo unido de Estados a través de una característica geográfica y de un grado de interdependencia mutua. En cuanto al regionalismo, se puede entender en relación no sólo a la institucionalización de las prácticas transfronterizas sino también, el reflejo de las transformaciones del espacio regional (Riggirozzi \& Tussie, 2012). Por otro lado, para Fawcett (2004) la regionalización puede posibilitar la formación o configuración de regiones, lo que a su vez puede dar lugar a la aparición de grupos regionales, actores y organizaciones. Es decir, este autor entiende el regionalismo como un proyecto político, y la regionalización como el proceso espontáneo que acompaña el incremento de la interacción y la actividad al interior de la región, en el mismo sentido que Nye.

Ahora bien, la agenda regional trabajada influye en el modelo regional adoptado. Es Scharpf (1996) quien propone los conceptos de integración negativa y de integración positiva, los cuales también pueden referirse a un regionalismo negativo o positivo. La forma positiva manifiesta los efectos de las políticas de cooperación internacional de los Estados identificados con una misma región, que en tanto un nivel menor de regionidad que el que expresa la 
integración regional, contribuyen a profundizar los lazos de confianza y lealtad, vía la autonomía relacional, entre los actores regionales estatales y no estatales. La regionidad, como lo llama Perrota (2013) hace referencia al concepto de regionness, entendido como:

El proceso por el cual un área geográfica se transforma de un objeto pasivo a un sujeto activo, capaz de articular los intereses transnacionales de la región emergente. Por lo tanto, la regionalidad implica que una región puede ser una región "más o menos". El nivel de regionalidad puede tanto aumentar como disminuir ${ }^{1}$ (Hettne y Soderbaum, s.f., p. 12).

Por su lado, autonomía relacional es un concepto acuñado por Russell y Tokatlian, definida como: "la capacidad y disposición de un país para actuar independientemente y en colaboración con otros, en forma competente, comprometida y responsable" (Russell y Tokatlian, 2002; citados en Pelfini, 2012, p. 103). Comprendiendo al Estado como actor de cooperación en el escenario internacional, la autonomía relacional posee como objetivo "la preservación y ampliación de grados de libertad" y, "entiende que las prácticas, instituciones, e ideas se definen y desarrollan dentro de un marco de relaciones en las que "el otro", en vez de opuesto, comienza a ser parte integral de lo que uno es" (Russell y Tokatlian, 2002, p. 179).

Antes de proseguir, cabe aclarar que cuando se refiere al concepto de integración regional, se alude a la concepción del enfoque neofuncionalista del término, que poniendo el acento en su dimensión política, la entiende como "el proceso por el que los actores políticos en varios marcos nacionales distintos se persuaden a cambiar sus lealtades, expectativas y actividades políticas hacia un nuevo centro, cuyas instituciones poseen o piden jurisdicción sobre los Estados nacionales preexistentes" (Haas, 1958, p. 16; citado en Posada, 2005, pp. 251-252). Las instituciones supranacionales, a las cuales los Estados les ceden parte de su soberanía apostando a avanzar en el regionalismo, garantizan el cumplimiento de lo pactado entre los socios regionales en búsqueda de beneficios absolutos e intereses compartidos. En consonancia, el neoliberalismo o institucionalismo liberal entiende que el rol que juegan las instituciones en un sistema internacional anárquico y con mercados imperfectos es crucial para impactar sobre el comportamiento de los Estados y limitar así las consecuencias de un sistema anárquico, dando certezas acerca de los acuerdos regionales y los resultados esperados (Keohane, 1993).

Sanahuja (2012) también plantea la idea de "agenda regional positiva" refiriéndose a la creación de instituciones y políticas comunes, y a una cooperación más intensa en ámbitos no comerciales. Una agenda regional positiva implica, además de la eliminación de obstáculos (como la baja de aranceles a los productos exportables o la libre circulación de las fuerzas de trabajo entre los socios regionales), una coordinación regional de políticas que regule y distribuya los beneficios alentando a los socios a pensar en beneficios absolutos más que en términos relativos.

Entonces, la cooperación se puede entender como un tipo específico de regionalismo que responde, en términos generales, tanto a una agenda con carácter positivo o negativo. Como 
plantean Axelrod y Keohane (1985), se asume que este regionalismo es posible en función de beneficios absolutos para los actores involucrados y de la mitigación de los efectos de la anarquía en un sistema internacional interdependiente, donde los efectos de las acciones de los actores poseen un efecto búmeran. Pero, asimismo se tiende a obstaculizar dada la inexistencia de órganos superiores que garanticen los acuerdos. De esta manera, el institucionalismo neoliberal explica que las instituciones internacionales y los regímenes ejercen efectos sobre el comportamiento de los Estados y limitan las consecuencias de un sistema anárquico, dando certezas acerca del cumplimiento de lo pactado (Keohane, 1993). Así, según los teórico neoliberales de las relaciones internacionales, la cooperación ocurre cuando las políticas llevadas a cabo por el gobierno de turno son percibidas por sus socios como estimulantes para la realización de sus propios objetivos, como resultado de un proceso de coordinación de políticas.

\subsection{De la Cooperación Sur-Sur}

A partir de los postulados mencionados, se puede sostener que en la medida que aumenta la autonomía relacional, la cooperación regional le va abriendo camino a la integración en tanto tipos específicos de regionalismo. Sin embargo, dada una relativamente baja autonomía relacional, se podría esperar que la cooperación sea contingente, y por lo tanto, que exista una escasa institucionalidad. En este sentido, en tanto coordinación regional de políticas entre países en desarrollo, la especificidad de la Cooperación Sur-Sur radica en brindar sólidas posibilidades para suavizar las asimetrías existentes entre los socios, a través de la elaboración conjunta y consensuada de políticas públicas en áreas funcionales y, en búsqueda de institucionalidad (supranacionalidad). Entonces, la CSS da cuenta de poseer características que Nye (1990) le otorga al softpower o "poder cooptivo/blando" definido como:

La capacidad de una nación de estructurar una situación de forma tal que las otras desarrollen preferencias o definan sus intereses de forma coherente con aquella nación. Este tipo de poder tiende a surgir de recursos tales como la atracción cultural e ideológica, tanto como de las reglas e instituciones de los regímenes internacionales (Nye, 1991, p. 184; citado en Colacrai, 2001, p. 37).

Ello significa que los Estados-Nación involucrados en calidad de socios en iniciativas de CSS procuran a través de acciones cooperativas horizontales extender su influencia y cooptar el apoyo de los gobiernos para determinados intereses propios, en lugar de utilizar la coacción. Según el Sistema Económico Latinoamericano y del Caribe (SELA), la CSS se plasma en relaciones directas y horizontales entre países que enfrentan problemas comunes y que tienen como propósito superar, a partir de esfuerzos conjuntos, los desafíos del desarrollo. La CSS se caracteriza y diferencia de la cooperación tradicional (Sagasti y Prada, 2011) por sus principios básicos de solidaridad, complementariedad, igualdad, no condicionalidad y respeto de la 
soberanía, los cuales crean una conciencia cooperativa (Lechini, 2009). Así, la CSS es una modalidad de la Cooperación Internacional al Desarrollo, la cual se aborda como:

Un conjunto de actuaciones, realizadas por actores públicos y privados, entre países de diferente nivel de renta con el propósito de promover el progreso económico y social de los países del sur de modo que sea más equilibrado en relación con el Norte y resulte sostenible (Gómez \& Sanahuja, 1999, p. 17).

Paralelamente, la CSS "comprende a aquellas acciones gestadas desde el gobierno central ("top-down") o desde subunidades estatales o locales ("bottom-up") orientadas a la promoción del desarrollo" (Malacalza, 2013, p. 14), ya sea a través de la asistencia técnica, créditos concesionales, apoyo a obras de infraestructura física, cooperación comercial, entre otros. La CSS asociada a acciones, proyectos o programas gestados "desde abajo", es decir, a partir de actores sub-nacionales, locales, de la sociedad civil y/o del sector privado, se puede asimilar a un proceso descentralizado de coordinación de políticas regionales. De esta manera, la CSS Descentralizada es una "cooperación que se caracteriza por la manera de compartir mutuamente conocimientos, habilidades, experiencia y recursos humanos, técnicos, económicos y/o financieros para alcanzar objetivos de desarrollo local o regional, a través de esfuerzos concertados entre los gobiernos y otros actores subnacionales" (SEGIB, 2017, p. 33). Los actores sub-nacionales incluyen así las comunidades, municipios, distritos, provincias, departamentos, regiones o estados federados y comprenden:

Los organismos públicos de gobierno directa o indirectamente elegidos a nivel subnacional, que cuentan -en un territorio concreto y de conformidad con la ley - con un grado de autonomía respecto del gobierno central así como con una serie de competencias para prestar servicios públicos a los ciudadanos (SEGIB, 2016, p. 42).

Los actores descentralizados poseen un valor insustituible ya que "aportan -0 deben aportar- al menos en dos dimensiones: la contribución a la articulación multinivel como elemento para la gobernanza global y la aportación de valor específico a los procesos de desarrollo" (Martínez y Sanahuja, 2012, p. 17). Dicho valor específico se encuentra relacionado a la participación de la ciudadanía local en el proceso de cooperación transfronteriza. Es en el marco de las relaciones entre territorios lideradas por sus gobiernos, es decir, por medio de mecanismos de coordinación de tipo "top-down", que otros actores locales poseen cierto grado (o no) de participación en el desarrollo de la cooperación descentralizada, como las universidades, empresas, sindicatos, organizaciones no gubernamentales, entre otros. La reciente conciencia de la comunidad internacional por el rol determinante que juegan los gobiernos sub-nacionales en los procesos de desarrollo de sus países y "la profundización en los procesos de descentralización y de nuevos paradigmas en materia de gobernanza que dan mayor protagonismo a la ciudadanía y a los operadores del territorio" (SEGIB, 2016, p. 17) son los factores principales del actual auge de esta modalidad de cooperación. 
Desde la reflexión teórica, se analiza la cooperación descentralizada, en tanto transfronteriza y se concibe a las fronteras como áreas estratégicas para los proyectos regionales, donde los gobiernos sub-nacionales y otros actores estatales y no estatales tienen un papel principal. En la frontera, principalmente entendida como espacio interestatal, se condensan las relaciones entre la población local y los gobiernos nacionales y regionales y/o locales, al mismo tiempo que se enfatizan las desigualdades entre las poblaciones fronterizas y al interior de las mismas (Desiderá Neto \& Penha, 2017). Esta modalidad de cooperación:

Es «una colaboración entre autoridades subnacionales más allá de los límites fronterizos nacionales» que permite la participación y actuación conjunta y en forma de red de los actores públicos y privados del territorio a ambos lados de la frontera (...) Su objetivo global es que las relaciones entre poderes territoriales vecinos se desarrollen con la misma naturalidad que si la frontera no existiese (Rhi-Sausi \& Oddone, 2010, p. 37).

$Y$ se basa en principios tales como: subsidiariedad, flexibilidad, proporcionalidad, participación ciudadana y transparencia administrativa y publicidad de los actos de gobierno (Rhi-Sausi \& Oddone, 2013). Es decir, su accionar desdibuja los límites del territorio nacional incentivando la coordinación de políticas regionales a nivel local:

Los procesos de integración regional provienen de la interacción de dos dimensiones complementarias: una dimensión que parte desde arriba, promovida por las instituciones nacionales, sobre todo a su más alto nivel, presidentes o jefes de gobierno, y una que parte desde abajo donde los actores principales son las instituciones subnacionales, gobiernos intermedios y locales, y los actores políticos, sociales y económicos de los territorios. La consolidación de estas dos dimensiones posibilita avances en la integración regional. La dimensión local es fundamental para gobernar los procesos de integración desde abajo, como los que se verifican cotidianamente en los espacios fronterizos (Oddone, 2017, p. 98).

La CSS Descentralizada se caracteriza también por ser selectiva e indirecta, orientadas a crear oportunidades para los diferentes agentes locales y un entorno territorial favorable al desarrollo inclusivo buscando conectarse con las políticas nacionales, regionales e internacionales (Alburquerque, 2001; Rhi-Sausi, 2008). Esto hace alusión a formas de gobernanza de la CSS menos jerárquicas y centralizadas, y a la necesidad de que existan instituciones específicas para desarrollar las acciones, proyectos y programas de CSS Descentralizada. En este sentido, es vital comprender la importancia del desarrollo de la paradiplomacia, principalmente porque "los municipios son la base de la vida democrática de la región en donde la elección directa de los intendentes o alcaldes contribuye a que la representación local sea legitimada por medio de los canales de participación creados" (Oddone, 2017, p. 99). Asimismo, también porque las unidades sub-nacionales se enfrentan cotidianamente a "movimientos" que pueden impulsar o hacer retroceder el modelo de regionalismo adoptado: "cada actor, cada escala local, es el punto de intersección entre fuerzas, y por consiguiente un punto de potencial resistencia a cualquier modo de actuar o pensar, o un 
punto de organización y promulgación de programas diferentes u opuestos" (Rose y Miller, 1992, p. 192).

Cuando se refiere a gobernanza, se hace alusión a un medio para conseguir una variedad de objetivos que son escogidos independientemente por los actores implicados y afectados (Schmitter, 2001; Prats Cabrera, 2003). Si bien la gobernanza hace referencia a las relaciones entre el Estado y los actores no gubernamentales, al gobierno como interacción (Kooiman, 2003), existen autores que resaltan la capacidad del gobierno de un Estado para dirigir, como lo son Peters (1998) y Pierre (2000). En otras palabras, "la afirmación de la evolución de gobierno a gobernanza proclama un cambio en el enfoque analítico que abarca desde las instituciones formales del gobierno hasta los procesos tanto formales como informales del gobierno de la sociedad y la economía" (Torfing \& Díaz-Gibson, 2016, p. 101).

Para estimular formas de co-gobernanza, ${ }^{2}$ la construcción de una institucionalidad para las microrregiones fronterizas constituye una condición indispensable (Oddone, 2017). Las zonas fronterizas implicadas en procesos de cooperación y/o integración necesitan esquemas de funcionamiento que permitan un elevado grado de participación social (gobernanza horizontal) y que condensen la interacción entre los diferentes niveles institucionales (gobernanza vertical), es decir, lo que se denomina conceptualmente como gobernanza multinivel:

La relocalización y desagregación de la autoridad gubernamental, antes centrada en el Estado, hacia otros niveles en una dimensión vertical -hacia arriba en las corporaciones, organismos y regímenes internacionales; y hacia abajo en los actores subnacionales- y horizontal; de manera que describe un sistema de negociación continua entre gobiernos de varios niveles territoriales (Chacón, 2017, p. 26).

En definitiva, la CSS Descentralizada es una modalidad de la Cooperación Internacional al Desarrollo que implica, a priori, relaciones de carácter horizontal entre países en desarrollo. Esta brinda a los bloques sub-regionales, como por ejemplo, el Mercosur, una oportunidad única para conformar y/o vigorizar instituciones supranacionales que garanticen el proceso regional, a los fines de lograr la convergencia de las estrategias de desarrollo y suplir las desigualdades sociales.

\section{LA CSS DESCENTRALIZADA EN LA REGIÓN LATINOAMERICANA}

El origen moderno de la cooperación descentralizada se puede ubicar a principios del siglo XX con la creación de la Asociación Internacional de Autoridades Locales (International Union of Local Authorities - IULA, en inglés) con sede en la Haya (Países Bajos). A finales de la década del 50 , en un escenario de descolonización y con un componente solidario-asistencialista, la cooperación descentralizada se asocia a las nuevas relaciones de las ciudades europeas con sus excolonias africanas, asiáticas y latinoamericanas. En 1957, se funda la Federación Mundial de Ciudades Unidas (FMCU) con el objetivo de impulsar los hermanamientos entre estas 
ciudades, que junto a la IULA, fueron los embriones de lo que hoy se conoce como Ciudades y Gobiernos Locales Unidos (CGLU), creada en 2004.

Hacia finales de la década del 60, Brasil comienza a firmar acuerdos de hermanamiento con ciudades africanas de habla portuguesa. Los hermanamientos entre Luanda (Angola) y Belo Horizonte y Brasilia se firman en 1968 y pueden ser considerados como las primeras iniciativas latinoamericanas de Cooperación Sur-Sur Descentralizada. El componente cultural y políticoideológico de esta modalidad de cooperación estuvo presente desde sus inicios. Por ejemplo, en la década del 70 y del 80 , ciudades europeas y canadienses establecen relaciones con ciudades de países en los que se han producido procesos revolucionarios, como Nicaragua, El Salvador y Cuba (SEGIB, 2016).

El primer documento internacional que introduce el concepto de cooperación descentralizada es Acuerdos de Lomé (1989), que rige las relaciones de cooperación entre 12 países miembros de la Comunidad Europea y más de 60 países subdesarrollados del conjunto llamado ACP (África, Caribe y Pacífico). Solamente un país hispano-parlante se encontraba entre los países ACP: Guinea Ecuatorial, si bien varios países anglófonos de América ya formaban parte del mismo, como Belice, Guyana y una serie de pequeños Estados del Caribe. La aproximación al concepto de cooperación descentralizada en este documento hace referencia a las relaciones de cooperación desarrolladas por todos aquellos agentes e instituciones que no forman parte del gobierno central, sean de carácter públicos o privados, para lograr un desarrollo en el que participe la población local. Respecto a América Latina:

La Comisión Europea empieza a lanzar toda una serie de programas horizontales de cooperación económica dirigidos a actores específicos: ALFA4 y ALBAN5 en el terreno de la educación; AL-INVEST6 en el terreno de la cooperación empresarial y tecnológica; @LIS7 en el terreno de las tecnologías de la información y la comunicación; ALURE en el ámbito de la energía, y URBAL en el de la cooperación entre gobiernos locales (SEGIB, 2016, p. 14).

Como queda en evidencia, el carácter económico-financiero con el que nace la cooperación descentralizada adquiere notoriedad a partir de la década del 90, y específicamente a partir de 1998, cuando el Consejo de la Unión Europea adopta un Reglamento sobre ella reconociéndola como una modalidad de la CID. Con la llegada del nuevo milenio esta perspectiva asistencialista se renueva, motivando la construcción de relaciones horizontales entre los socios implicados. Si bien la cooperación descentralizada tradicional y la Sur-Sur atienden problemáticas similares y responden a una misma lógica de base, los ejes temáticos priorizados y las formas de gobernanza varían entre las relaciones Norte-Sur y Sur-Sur. La horizontalidad de la CSS Descentralizada adquiere mayor relevancia al ser un principio básico en su accionar, y a su vez, la articulación en calidad de socios trata de limar las asimetrías existentes entre las ciudades de esos países. En cuanto a las temáticas que viene trabajando la CSS Descentralizada, la mayoría de las iniciativas se asocian a la dimensión social como 
económica del proceso de coordinación de políticas regionales. Entre los ejes se destacan: medio ambiente y energía, educación y formación profesional, e infraestructura (SEGIB, 2016).

En los últimos años, los gobiernos sub-nacionales latinoamericanos han adquirido un notorio dinamismo en el escenario de la cooperación, tanto a escala bilateral, como a través de redes de ciudades; ejemplo de ello es Mercociudades, en el marco del proyecto regional del Mercosur (esta iniciativa se tratará más adelante). En este sentido, los procesos de descentralización en la coordinación regional de políticas llevados a cabo en Argentina, Brasil o México han sido muy significativos para otros países en desarrollo. Actualmente, hay ciudades especialmente dinámicas como Medellín (Colombia) y Sao Paulo y Belo Horizonte (Brasil), que han establecido relaciones tanto con ciudades de la región como con europeas y africanas.

Los gobiernos sub-nacionales argentinos, brasileños y mexicanos disponen de un peso político y de recursos importantes desde el punto de vista de las capacidades técnicas. Estos reciben el apoyo técnico de los gobiernos centrales a través de estructuras específicas, a veces insertadas en las Cancillerías o en las agencias nacionales de cooperación, o bien, en instancias como secretarías o direcciones en Ministerios. En estos esquemas, los gobiernos nacionales latinoamericanos apuntan la necesidad de que la CSS Descentralizada se alinee con las estrategias y las prioridades marcadas a nivel nacional en materia de relaciones exteriores y cooperación internacional. Sin embargo, gran parte de los países de la región poseen formas de gobernanza más bien centralizadas y jerárquicas que escapan de los mecanismos de tipo "botton up" (SEGIB, 2016).

La existencia de algún marco legal y/o normativo que determine la autonomía de actividades de la gestión de la cooperación por parte de gobiernos subnacionales es de vital importancia para analizar las formas de gobernanza que se registran y determinar si la CSS Descentraliza incurre en las mismas contradicciones que la cooperación tradicional, es decir, estudiar los mecanismos institucionales y sus funcionamientos sirven para determinar las "enfermedades" de esta cooperación. Para 2016, según una encuesta realizada por el Programa Iberoamericano para el Fortalecimiento de la Cooperación Sur-Sur (PIFCSS) a 17 países iberoamericanos, solamente 9 (Argentina, Colombia, Ecuador, El Salvador, España, Perú, Portugal, República Dominicana, y Uruguay) poseían algún tipo de regulación a la cooperación descentralizada y en tan solo 2 (Argentina y Ecuador) la acción exterior de los gobiernos subnacionales tiene reconocimiento constitucional. ${ }^{3}$ Resulta por lo menos llamativo, que siendo Brasil el "fundador" de la CSS Descentralizada sea uno de los países en el cual aquella no se encuentra regulada.

Particularmente para el sub-campo de la CSS en el sector educativo, Surasky (2013) plantea que en Latinoamérica, esta atiende a demandas autóctonas de los países socios motivados por la autoafirmación de las relaciones Sur-Sur, a través del desarrollo de una conciencia de sus capacidades y fortalezas. En la misma línea argumental, Perrota (2015) afirma que esta cooperación es parte de una agenda social más amplia de coordinación regional de 
políticas en un escenario global, que responde a profundizar las relaciones intra-regionales. Ello genera efectos directos e indirectos sobre la construcción de un regionalismo positivo a través de iniciativas de carácter técnico (Milani, 2015).

Según la SEGIB (2016), en gran parte de los países de América Latina, las necesidades de la CSS educativa se dirige a mejorar aquellos aspectos que vinculan la educación con la incorporación posterior del alumnado, bien en el mercado laboral o en actividades de emprendimiento. De esta manera, la CSS se constituye como una herramienta para anclar el proceso regional desde una agenda positiva (Perrota, 2012), y a su vez, dadas las incidencias del campo educativo, amarra el regionalismo a los valores comunes y a la afirmación identitaria (Pinheiro \& Beshara, 2011) vigorizando la autonomía relacional del Estado-Nación, y reclamando cohesión social (Di Filippo \& Franco, 1999). Es decir, la CSS en educación viene a afianzar las capacidades nacionales desde un prisma regional.

Particularmente, a través de la CSS Descentralizada en educación, la región internaliza la dimensión territorial en el diseño de las políticas públicas, a partir de la inclusión como variable estratégica para una interacción intersocietal sustentable en los estudios vinculados a esta temática. En este sentido, la cooperación orientada al "apoyo a la gobernabilidad local, hacia el fortalecimiento de la sociedad civil y las instituciones locales, o hacia el apoyo al diseño y la gestión de políticas públicas en el ámbito local" (Martínez y Santander, 2009, pp. 55-56; Illán, 2006; citados en Martínez y Sanahuja, 2012, pp. 18-19) ayudaría a la planificación y focalización de la gestión de las políticas de cooperación en esta área de la agenda regional.

En otras palabras, la relación existente entre las políticas nacionales de educación y la coordinación regional de las políticas educativas puede concebirse como una articulación que se refuerza y legitima de forma bidireccional a los fines de mejorar la calidad de vida de la población en su conjunto; es decir, la política regional se "construye y se afirma a partir de las políticas nacionales (en especial a partir de la cooperación y coordinación entre los Estados Parte) y, al mismo tiempo, el nivel regional refuerza y legitima el nivel nacional" (Perrota, 2012, p. 16). El auge y repolitización del debate sobre el regionalismo latinoamericano, se viene desarrollando en paralelo con "la legitimación y el fortalecimiento de las capacidades de los gobiernos subnacionales para actuar en el plano internacional (paradiplomacia) con el fin de favorecer un desarrollo local transfronterizo articulado con la integración regional (place branding)" (Odonne, 2017, p. 94) vertebrándose así uno de los argumentos principales para la cooperación descentralizada de las últimas dos décadas en el Mercosur.

\subsection{CSS Descentralizada y la agenda educativa del Mercosur}

Se considera que el universo de la Cooperación Sur-Sur en sectores sociales engloba más que un amplio abanico temático que requiere expertises particulares. En relación a la construcción y consolidación del Estado-Nación y, concibiendo la educación como un proceso de adquisición de conocimiento, la CSS latinoamericana en áreas sociales se implementa a los 
fines de sortear los retos del proceso de desarrollo que enfrentan los países con un bajo o mediano nivel de renta (Milani, 2015; Steiner-Khamsi, 2009). En este sentido, en el marco del Consenso de Buenos Aires, ${ }^{4}$ con un esquema de regionalismo de carácter post-liberal (Sanahuja, 2012) y al institucionalizarse esta modalidad de cooperación en el Mercosur, los socios comienzan a atender nuevas temáticas vinculadas a la dimención social del proceso regional.

En los comunicados oficiales del Mercosur se planteaba que enfatizar la dimensión social del proceso regional podría impulsar la educación como "componente fundamental de las políticas de desarrollo humano sostenible con equidad social, que permita superar la pobreza y la marginación de amplios sectores de nuestros pueblos, fortaleciendo el desarrollo productivo, científico y tecnológico, y la democratización de conocimientos y saberes" (Boletín Mercosur Especial, 2010, p. 4). La promoción de la cooperación inter-institucional entre instituciones educativas del nivel superior de los países socios, vinculada a la formación de docentes universitarios, es históricamente el área de la agenda de cooperación más trabajada por el Mercosur.

En sus inicios, la cooperación descentralizada en las fronteras del Mercosur estuvo asociada a solucionar problemáticas de índole comercial (como la libre circulación de bienes), por lo que se asociaba a una agenda regional negativa. Asimismo, la articulación de los territorios fronterizos con los programas del Fondo para la Convergencia Estructural del Mercosur (FOCEM), les permitía a las unidades sub-nacionales movilizar recursos para alcanzar objetivos concretos, aunque no existían fondos estructurales dedicados exclusivamente a las zonas de frontera con el objetivo de construir "nuevos núcleos de desarrollo" (Oddone, 2017, p. 97).

En la Cumbre de Belo Horizonte de diciembre de 2004, el Consejo Mercado Común (CMC) resolvió crear el Foro Consultivo de Municipios, Estados Federados, Provincias y Departamentos del MERCOSUR (FCCR), el cual se integra con representantes de las diferentes instancias locales y regionales designados por los Estados Partes. La Decisión N41/04 establece que su objetivo es "estimular el diálogo y la cooperación entre las autoridades de nivel municipal, estadual, provincial y departamental dos Estados Partes del MERCOSUR". En el artículo cuarto de la misma se indican las potestades: "podrá proponer medidas destinadas a la coordinación de políticas para promover el bienestar y mejorar la calidad de vida de los habitantes de los Municipios, Estados Federados, Provincias y Departamentos de la región, así como formular recomendaciones por intermedio del Grupo Mercado Común".

El Reglamento Interno del FCCR fue aprobado tres años más tarde por Resolución $N^{\circ}$ 26/07 del Grupo Mercado Común (GMC). En el artículo primero se destaca que el FCCR "es el órgano de representación de los gobiernos locales de los Estados Parte del MERCOSUR" y en el tercer artículo que "estará conformado por un Comité de los Municipios y un Comité de los Estados Federados, Provincias y Departamentos". A partir de sus Comités, el Foro debería 
propiciar una visión compartida sobre el desarrollo territorial en el marco del proceso regional, favorececiendo un desarrollo equilibrado con sostenibilidad ambiental y cohesión social que contemple las particularidades y necesidades de las zonas de frontera del bloque.

Dentro del primer Plan de Acción 2013-2014 del FCCR (aprobado por la Decisión CMC № 54/12), se priorizaron las siguientes estrategias: 1) articular acciones de cooperación entre los actores subnacionales y locales de frontera; 2) apoyar el desarrollo de legislación de fronteras e incentivar su implementación; 3) reactivar el Grupo de Trabajo de Integración Fronteriza; 4) definir la agenda estratégica del FOCEM para áreas de frontera; 5) acompañar y validar el proyecto de cooperación en materia de gobernanza fronteriza con la AECID; y, 6) crear oportunidades para el desarrollo integrado de políticas públicas para las comunidades tradicionales y afrodescendientes en áreas de frontera. Al respecto de la institucionalización de la CSS Descentralizada en el marco del SEM, el proceso de afianzó con:

La creación del Grupo Ad Hoc sobre Integración Fronteriza (GAHIF), vinculado al Grupo Mercado Común (GMC). Coordinado por el Ministerio de Relaciones Exteriores (MRE) e integrado a los órganos técnicos responsables de los temas relacionados con la agenda, el GAHIF tenía como objetivo crear instrumentos normativos $u$ otros cursos que promovían la integración de las comunidades fronterizas, con miras a la mejora de la calidad de vida de esas poblaciones sin perjuicio para los regímenes nacionales o los negociados entre dos o tres Estados Partes ${ }^{5}$ (Desiderá Neto \& Penha, 2017, p. 204).

La CSS Descentralizada en educación se vinculaba principalmente a la dimención interna del proceso regional: a la coordinación de políticas entre los países Mercosur más que a la cooperación extra-bloque. Sin embargo, también se realizaron proyectos conjuntos de carácter técnico con España e Italia por ejemplo, así como con la Comunidad Europea. De esta manera, las relaciones extra-bloque desarrolladas por el SEM evidencian que la cooperación tradicional era pensada como complementaria a la CSS en educación.

La institución escolar es considerada el espacio por excelencia donde se desarrolla el encuentro entre las poblaciones de frontera y en el cual se ponen en evidencia todo tipo de desigualdades, incluso las tensiones interculturales que obstaculizan la inclusión educativa. ${ }^{6}$ Asociada a ella se encuentran las problemáticas lingüísticas, que involucran a las poblaciones indígenas y también al alumnado migrante en zonas fronterizas. Así se acordó, por ejemplo, que las actividades del Proyecto Caminos del Mercosur, ${ }^{7}$ se puedan realizar en español, guaraní y portugués. Este Proyecto se implementó desde el 2003, con el objetivo de fortalecer la identidad mercosureña de los jóvenes de 16 y 17 años de la región, por medio de una experiencia formativa en la que los participantes, además de ampliar sus conocimientos, desarrollen una vivencia que permita apreciar el valor de la integración regional, respetando la diversidad cultural.

En cuanto a la cuestión lingüística, principalmente asociada a la educación básica, el grupo de trabajo de Políticas Lingüísticas (GT-PL) tuvo un rol fundamental. La agenda del CAPL poseía como eje temático la construcción de una una política lingüística regional, a través de diferentes 
dispositivos de comunicación y de espacios de debate entre los países involucrados, como así también entre las propias unidades del SEM (mecanismos de coordinación intra-institucional). EI SEM expresaba la preocupación por la pérdida de la riqueza lingüística regional, ya que su efecto sería inversamente proporcional para el grado de peligro de convertirse en "consumidores de programas ajenos" (GT-PL ACTA N01/08).

Las problemáticas lingüísticas históricamente han sido un desafío para lograr los resultados esperados de la cooperación transfronteriza entre los socios del bloque. Como propuesta a esta preocupación, se planteaba reflexionar sobre la legislación de cada país Mercosur. Las lenguas indígenas eran consideradas lenguas extranjeras, por lo que era imperioso considerar cada situación sociolingüística. En este sentido, la formación docente y su movilidad se presentaban como las herramientas clave para generar un proceso de toma de conciencia de la problemática lingüística regional. Asimismo, el GT-PL planteaba que desde el nacimiento del Mercosur, la formación docente vinculada a escuelas de frontera se basó en la defensa de la nacionalidad y en un concepto moderno de la frontera. Por lo que era necesario modificar esta concepción y vincularla a la noción de identidad Mercosur.

Para incentivar y fortalecer la ciudadanía regional a partir de iniciativas bilaterales, el SEM afirmaba que era necesario articular las actividades del GT-PL con las acciones y políticas a nivel nacional, a través de la coordinación con los Ministerios de Educación, las unidades subnacionales y con participación ciudadana. Tanto el FOCEM, como el Foro Consultivo de Municipios, Estados Federados, Provincias e Intendencias del Mercosur (FCCR) fueron, desde su creación, espacios de manifestación de los gobiernos sub-nacionales. En este contexto nace en 2005 el Proyecto "Escuelas Bilingües de Frontera" (PEBF). ${ }^{8}$ El Programa comienza siendo una iniciativa bilateral de Brasil y Argentina, a los fines de desarrollar un modelo de enseñanza común en las escuelas de frontera para el nivel primario -y también pre-escolar en el caso argentino-, y garantizando que alumnos y docentes tengan la oportunidad de educarse y comunicarse en portugués y español a partir del desarrollo de un programa intercultural. Al respecto, "es muy común que dentro de los procesos de integración regional "se bilateralicen" los acuerdos en materia de cooperación transfronteriza" (Rhi-Sausi \& Oddone, 2013, p. 275).

Ya en 2010, Argentina y Brasil manifestaban que tenían problemas de gestión financiera para la realización de los intercambios docentes, pero pretendían extender el PEBF a Paraguay y a Uruguay con financiamiento del FOCEM (CCR/CRC-EB/GT ESCUELA DE FRONTERA/ACTA $N^{\circ}$ 01/10). El PEBF generó oportunidad es de superar la idea de frontera nacional como una barrera visualizando el acceso a oportunidades sociales, personales, educativas, culturales, y económicas nacidas de la presencia y en la interacción con el otro (Documento Escuelas de Frontera - Marco Referencial de Desarrollo Curricular). Se definió como lugar privilegiado para el desarrollo del Programa el sistema de ciudades gemelas internacionales, las cuales se identifican con las ciudades que cuentan con otra cercana y colega en el otro país, promoviendo las condiciones para la cooperación transfronteriza. El PEBF 
basaba el intercambio docente a partir de la disponibilidad de planteles ya formados en ambos países actuando en las escuelas involucradas. La metodología utilizada, de la enseñanza vía proyectos de aprendizaje (EPA) permitió que se elijan los proyectos a ser desarrollados localmente, por grupo o por escuela, de acuerdo con lo que se considere más oportuno y de acuerdo con las diferentes realidades de las escuelas en cuestión:

Las primeras clases de la docente oriunda de la escuela-espejo tienen, por lo tanto, la doble función de permitir a la docente la familiaridad con sus nuevos alumnos y el relevamiento de intereses que servirán de base al primer proyecto de aprendizaje. A consecuencia de esta práctica, la planificación inicial -aquella que se realiza en el momento que el docente comienza a trabajar con el nuevo grupo- es de corto plazo (planificación clase a clase) y enseguida, una vez establecida la problemática del primer proyecto de aprendizaje a realizarse, se pasa a una planificación de mayor aliento (planificación de mediano y largo plazo). La planificación conjunta es uno de los momentos en los cuales el docente cuenta con el asesoramiento pedagógico local, ofrecido por la escuela o por los ministerios (Documento Escuelas de Frontera - Marco Referencial de Desarrollo Curricular, s/f, p. 29).

Como se mencionó anteriormente, el PEBF presentaba dificultades presupuestarias, y los cambios de las autoridades políticas y la congestión de la comunicación entre las unidades subnacionales y el Ejecutivo (CCR/CRC-EB/GT ESCUELA DE FRONTERA/ACTA № 01/13), fueron todas variables que obstaculizaban el avance del Programa. Sin embargo, los actores locales buscaron movilidades alternativas entre las escuelas para incentivar el intercambio docente y continuar con las actividades. A través de esta "bilateralización" de la agenda regional educativa, se evidenciaba que la cooperación descentralizada/transfronteriza comenzaba a mostrar indicios de mecanismos de gobernanza multinivel (horizontal y vertical) acompañada por la participación de la población local. Ello sobre el colchón de características comunes compartidas de forma intencionada:

Se puede decir que existe una integración cotidiana y espontánea de frontera. Al mismo tiempo, los procesos de integración, impulsados por la administración central de los países, también influencian al conjunto de la sociedad en el ámbito rutinario, ya que impactan unidades gubernamentales estatales y locales. La participación de los gobiernos subnacionales en esas relaciones internacionales -la paradiplomacia- lleva a la descentralización de las iniciativas externas del país, reuniendo a diversos agentes en una red de gestión más sensible y cercana a los problemas cotidianos de territorios marginados. Los acuerdos paradiplomáticos actúan por medio de contactos permanentes o ad hoc, con entidades públicas o privadas extranjeras, a fin de promover actividades socioeconómicas y culturales $^{9}$ (Benzatto e Prado, 2014; en Desiderá Neto \& Penha, 2017, p. 208-209).

Como plantea Oddone (2017), esta regionalización en zonas de frontera a partir del sector educativo necesita de un proceso de institucionalización específico para afianzar el regionalismo y lograr mayores grados de regionidad. Para Rhi-Sausi \& Oddone (2013), el fortalecimiento institucional del tejido comunitario implica la existencia de un marco jurídico que permita la acción consensuada de las unidades sub-nacionales. A causa de la multidimensionalidad de la 
temática fronteriza, las competencias en la materia se encuentren desagregadas en diferentes niveles e instituciones. Por ello el SEM resaltaba la importancia de mantener una vinculación permanente entre los Equipos de Escuelas de Frontera y los del GT-PL, para la articulación y compatibilización de las actividades al interior de cada socio a través de la cooperación transfronteriza. Paralelamente, el GT-PL se preocupaba por la participación continua de las delegaciones de países, ya que muchas de ellas se ausentaban en las reuniones de los diversos grupos; situación que obstaculizaba la coordinación de políticas regionales. Asimismo, el CAPL reconocía la necesidad de trabajar de manera conjunta con Comisión Regional Coordinadora Educación Superior y el Grupo de Trabajo Escuelas de Frontera.

A los fines de impulsar la coordinación regional de políticas desde abajo, la paradiplomacia es de vital importancia ya que brinda un marco institucional a la regionalización natural que se desarrolla en zonas de frontera, a través del establecimiento de contactos permanentes o ad hoc con entidades públicas o privadas extranjeras (Cornago, 2001; citado en Rhi-Sausi \& Oddone, 2013). El rol de la paradiplomacia en el caso del Mercosur se evidencia, por ejemplo, en la creación de las Mercociudades (Gomes, 2017), definida como una red que trabaja por unidades temáticas siendo una de ellas la educativa. Así, se incentiva la creación de redes de coordinación de políticas entre los gobiernos locales, que implementadas por las instituciones del territorio llaman a la participación de los otros actores locales promoviendo mayor articulación y generando mayores posibilidades de gestión compartida. De esta manera, los gobiernos locales poseen:

Mejores condiciones para exponer sus demandas a los gobiernos centrales responsables de la conducción del bloque regional. Con ello, la Red les ofrece la vía adecuada para concretar la integración fronteriza, pues fortalece los lazos políticos y viabiliza acciones reales de desarrollo de los territorios vecinos ${ }^{10}$ (Gomes, 2017, p. 12).

En 2009, se crea el Grupo de Trabajo en Cooperación Descentralizada de Mercociudades (GTCD) que en asociación con el Observatorio de la Cooperación Descentralizada Unión Europea/América Latina (OCD), buscaba "articular acciones de formación continua de gestores locales, de circulación del patrimonio intelectual que en la materia posee, como así también la búsqueda de elementos que permitan analizar y apuntalar la internacionalización de los gobiernos locales y regionales" (Informe GCD 2009/2010, p. 1). ${ }^{11}$ Asimismo, en septiembre de 2015, la Unidad Técnica de Integración Fronteriza de las Mercociudades -creada en 2013propone la creación de "Mercorregiones", como espacios de cooperación transfronteriza referentes a mejorar los servicios públicos en estas zonas a través de consorcios públicos.

Por otro lado, también se desarrolló el Proyecto "Integración Fronteriza del Mercosur" (2008-2011), en el marco del Grupo de Trabajo de Integración Fronteriza, y con apoyo financiero de la Agencia Española de Cooperación Internacional para el Desarrollo (AECID). La contraparte por el Mercosur fue el FCCR, y tuvo como objetivo "diseñar metodologías de trabajo que sirvan de insumo para el FCCR y que faciliten las políticas públicas locales de integración fronteriza a 
partir de un diagnóstico de problemáticas en los territorios" (Programa Mercosur de la AECID, 2011 , p. 27), con un presupuesto total de 759.926 euros (FCCR-CN/ACTA N ${ }^{\circ} 2 / 10$ ). Los objetivos específicos fueron, por un lado, la sistematización de los diagnósticos e investigaciones de las problemáticas de integración fronteriza; y por el otro, la consulta a los actores locales para la elaboración de propuestas en materia fronteriza dando prioridad a la integración productiva, la inclusión social y el tratamiento de asimetrías regionales. La iniciativa continuó durante 20112013 a través del Proyecto "Gobernanza Fronteriza. Fortalecimiento de las Capacidades de los Gobiernos Departamentales y Locales del Mercosur", centrado en cursos de capacitación para funcionarios de ciudades gemelas, con un financiamiento de la AECID de 412.764 euros.

Dadas las motivaciones de este trabajo, tampoco se puede dejar de mencionar el Proyecto "INNOVA" (2009), financiado por la Comunidad Europea, a los fines de fortalecer el protagonismo local en las iniciativas de cooperación, a través de la capacitación a autoridades del territorio y actores de la sociedad civil. De esta manera, se esperaba un aumento en la elaboración de proyectos de carácter regional financiados con fondos de cooperación internacional extra-bloque, que impulsen "la ciudadanía regional, la inclusión social, la participación, la equidad de género, la cooperación pública/privada y el desarrollo de políticas locales para alcanzar los Objetivos de Desarrollo del Milenio (ODM)" (Boletín Mercosur $\mathrm{N}^{\circ} 26$, 2009 , p. 5). INNOVA venía a impulsar a los actores subnacionales y a las organizaciones no gubernamentales en el marco de las Mercociudades.

También cabe mencionar que en 2015, se crea el Subgrupo de Trabajo №18 "Integración Fronteriza" (SGT N¹8) en articulación con otros ó rganos y foros que trabajan temas específicos relativos a la cuestión fronteriza y coordinados por el Consejo del Mercado Común, a los fines de tratar cuestiones relativas a salud, educación, trabajo, migración, transporte, infraestructura, desarrollo urbano, desarrollo económico, pueblos indígenas, cooperación, integración productiva (GMC/RES. N N 59/15), entre otras. La creación de este Subgrupo nuevamente brindó oportunidad para reactivar la propuesta brasileña de un Acuerdo para la Promoción de la Integración Fronteriza a los fines de crear las Mercorregiones.

A partir del cuarto artículo de la propuesta del Acuerdo se podría entender una interpretación de la cooperación transfronteriza para todo el Mercosur como cualquier acción concertada destinada a reforzar y promover relaciones de vecindad entre sus comunidades 0 autoridades territoriales sobre la jurisdicción de dos o más Partes y con la celebración de cualquier acuerdo o arreglo necesario para la consecución de tal fin. Asimismo se respalda la conformación formal tanto como la articulación funcional de las Mercorregiones impulsando la función de paradiplomacia transfronteriza para el establecimiento de los vínculos intraregionales.

Por último, se quiere destacar que actualmente se sigue incentivando la Cooperación SurSur en las fronteras del Mercosur a través de distintos mecanismos institucionales. Así, el Plan de Acción 2016-2017 del FCCR (aprobado por la Decisión CMC № 48/15) establece que para 
promover la inclusión social de los ciudadanos del Mercosur es necesario elaborar políticas públicas comunes en educación, salud, medio ambiente, seguridad ciudadana e igualdad de género, en vínculo con la identificación de cadenas productivas y economías solidarias regionales.

Asimismo, uno de los ejes estratégicos del nuevo Plan de Acción del SEM hasta 2020 (Decisión CMC N²1/17) es "incentivar la inclusión y participación social a partir de programas regionales en el área educativa" (objetivos estratégicos/ejes $N^{\circ} 4$ ) a través de la profundización del Parlamento Juvenil del Mercosur, Caminos del Mercosur y Escuelas Interculturales de Frontera. Por su lado, el Plan de Acción de la Reunión Ministros y Autoridades de Desarrollo Social del Mercosur para el período 2017-2018 (Decisión CMC № 15/17) establece el fortalecimiento de la CSS en zonas de frontera vinculada a temáticas como la pobreza y la protección integral de niños y niñas en la primera infancia. En el mismo sentido, la Decisión CMC $N^{\circ}$ 30/17 plantea en su artículo primero que la Unidad de Apoyo a la Participación Social (UPS) integrará la estructura de la Secretaría del Mercosur (SM), cuyos representantes "actuarán como un canal de diálogo con la sociedad civil y con los movimientos sociales" (art. tercero).

\section{REFLEXIONES FINALES}

Tras los 26 años de existencia del Mercosur, los principios básicos de la CSS han guiado la política de cooperación del bloque. Como una modalidad complementaria a la cooperación tradicional y a partir de un proyecto regional que ha priorizado la dimensión social para anclar la construcción de regionidad, la CSS ha demostrado ser un vehículo efectivo para profundizar un modelo regional "positivo" con la participación de los actores locales estatales y no estatales. En la mayoría de los casos, las políticas públicas latinoamericanas no responden a decisiones participativas, sino que ellas son el resultado de intercambios entre expertos situados en contextos diferentes a los ejecutores de las mismas; es decir, los actores que participan en el detalle del diseño de las políticas educativas son foráneos al terreno local, en el cual se desenvuelven demandas multidimensionales propias de las zonas de frontera (Durán Jara, 2008). En este sentido radica la especificidad de la descentralización de la CSS en zonas fronterizas, la cual abre nuevos mecanismos de participación de la ciudadanía local en el proceso de coordinación de políticas regionales. Especialmente en el caso del Sector Educativo del Mercosur, esta cooperación puede ser la clave para apoyar al Estado en la búsqueda de la eficacia de las políticas sociales locales, contribuyendo así al desarrollo socio-económico vía la educación inclusiva.

Es decir, la cooperación transfronteriza redimensiona las zonas de frontera a través del proceso regional, como espacios estratégicos y genuinos de coordinación regional de política en múltiples niveles, apoyando la convergencia socio-económica en la región, fundamental para un desarrollo balanceado a largo plazo. Esta coordinación incentiva a los países socios a sumar esfuerzos para construir un sistema regional de educación, arduo camino a recorrer para 
impulsar la convergencia regional de las estrategias de desarrollo. De esta manera, se puede pensar que la CSS Regional Descentralizada en educación posibilita la existencia de un doble engranaje que se retroalimentaría vigorosamente favoreciendo el regionalismo del Mercosur y el desarrollo social inclusivo desde el territorio fronterizo de los países socios. En este sentido, el CAPL ha tenido en los últimos años un rol fundamental vinculado a la construcción de ciudadanía regional, en apoyo al encuadre del proceso de regionalización a partir del sector educativo, y a favor del fortalecimiento de la autonomía relacional a partir de una agenda positiva de cooperación transfronteriza entre los socios del Mercosur.

Sin embargo, la escasa institucionalidad en las zonas de frontera del bloque marca la necesidad de que los países sigan esforzándose para concretar mecanismos de consenso entre los diferentes actores locales, estatales y no estatales. La CSS Regional Descentralizada enaltece el rol del Estado a través de sus unidades sub-nacionales, ya que las mismas pueden contribuir con una mirada estratégica a la formulación, planificación, implementación, supervisión y evaluación de las políticas y estrategias nacionales de desarrollo social. En paralelo, colaborar a que las acciones implementadas a partir de la cooperación se coordinen y desarrollen respondiendo a las prioridades de la población fronteriza. Para lograr los resultados deseados, según Rhi-Sausi \& Oddone (2013), la construcción de una institucionalidad específica y eficaz para las áreas fronterizas es trascendental ya que "la construcción de una institucionalidad para las áreas fronterizas, en el marco de los enfoques funcionalistas, constituye una condición sine qua non para la sostenibilidad de la cooperación territorial y la integración regional" (Oddone, 2017, p. 100).

Se puede establecer entonces, que la CSS Regional en su modalidad descentralizada incentiva desde el territorio la capacidad y disposición de los socios para actuar independientemente y en colaboración con otros. Particularmente en el caso del SEM, la competencia, el compromiso y la responsabilidad de los socios para construir un sistema regional de educación todavía son incipientes, cuyos esfuerzos se encuentran atados a la voluntad política de los gobernantes dado el carácter no vinculante de la institucionalidad del bloque. En el marco de un Mercosur post-liberal, durante el período 2003-2015, como plantea Oddone (2017), la escasa institucionalidad en las zonas de frontera ha obstaculizado la visibilización de la agenda territorial y el consenso entre el sector público, los actores privados y la comunidad epistémica del bloque, manifestando así la necesidad de una gobernanza multinivel. En este sentido, el rol de las unidades sub-nacionales para guiar el proceso de construcción de autonomía relacional desde el territorio, para la identificación de intereses complementarios a partir de las demandas locales, es fundamental para profundizar el grado de regionidad del Mercosur.

Las iniciativas, proyectos y programas desarrollados en el marco del Sector Educativo del Mercosur mencionados en este trabajo son una aproximación a los esfuerzos del bloque por fortalecer el proyecto regional, especialmente, desde una agenda positiva de CSS 
Descentralizada en zonas de frontera. En resumen, en el Mercosur pueden identificarse una serie de demandas sobre cooperación trasfronteriza en educación que encuentran su origen en problemáticas específicas que atañen a las poblaciones locales (Rhi-Sausi \& Odonne, 2013). En el marco de un regionalismo positivo, la agenda educativa regional ha marcado ciertos ejes temáticos para dar solución a los principales problemas de los sistemas educativos (la deserción, la repitencia y, la escasa actualización y formación docente), estos ejes son: mejorar la calidad educativa; articular la escuela con la comunidad; promover la inclusión educativa; formar a la ciudadanía y actualizar la currícula escolar (Unidad Temática de Educación de la Red Mercociudades, gestión/2014).

En el marco de un regionalismo latinoamericano que está dejando de lado su carácter "post-liberal" y con escenarios sociales y económicos nacionales menos estables, este trabajo es una primera aproximación a los desafíos y oportunidades que plantea la cooperación transfronteriza en el Mercosur en la actualidad, para impulsar desde la dimensión social, el proyecto regional a partir de las zonas de frontera. ¿Es esta modalidad de cooperación una manera más eficiente de abordar las relaciones entre socios? o bien ¿incurre en las mismas contradicciones en que ha caído la cooperación descentralizada tradicional, es decir, si sirve también para reproducir articulaciones de carácter desigual?. Son preguntas que abren una agenda futura de investigación especialmente vinculada a la coordinación regional de políticas educativas.

\section{Notas}

${ }^{1}$ Traducción propia.

2 Para Kooiman (2003) existen tres distintos tipos de gobierno: autogobierno (self-governing), cogobierno (co-governing) y gobierno jerárquico (hierarchical governing). El primero se refiere a las interacciones sociopolíticas fluidas e indireccionadas de las sociedades modernas, en las cuales los sectores se gobiernan con ciertas limitaciones. Las formas de co-gobernanza se centran en la horinzontalidad, es decir, las entidades que participan de la estructura de gobierno poseen algún grado de igualdad y son autónomas. Mientras que, en los modos jerárquicos de gobernanza, los derechos y obligaciones se organizan según responsabilidades y tareas supraordenadas y subordinadas.

3 Para mayor información se puede consultar el capítulo cuarto del Marco Normativo Iberoamericano sobre Cooperación Descentralizada de "La Cooperación Descentralizada en Iberoamérica. Una mirada desde la Cooperación Sur-Sur" Serie - Documentos de trabajo / DT11 de la Secretaría General Iberoamericana - Programa Iberoamericano para el Fortalecimiento de la Cooperación Sur-Sur.

${ }^{4}$ El Consenso de Buenos Aires es un documento que se firma el 16 de octubre de 2003 por los Presidentes de Argentina y Brasil, Néstor Kirchner y Lula da Silva. En este se expresa como objetivos fundamentales del proceso de integración latinoamericano, la autonomía y el desarrollo de la región. Así se reorienta el modelo de regionalismo adoptado para el Mercosur hacia una agenda que priorice el carácter positivo del proceso incluyendo una mayor participación de la ciudadanía y la sociedad civil en la coordinación de políticas.

${ }^{5}$ Traducción propia.

6 Cabe destacar que la inclusión educativa puede encuadrarse en diferentes perspectivas (Ainscow \& Miles, 2009) pero la postura más utilizada por los estudiosos de la temática se refiere a la atención a la diversidad, es decir, asegurar la igualdad de oportunidades y combatir la discriminación en la promoción de la equidad; además es pensada como un proceso de 
interminable búsqueda de formas adecuadas de responder a las problemáticas vinculadas a la interculturalidad (Rico, 2017).

${ }^{7}$ Para mayor información, se puede visitar el sitio web oficial: http://www.edu.mercosur.int/esES/programas-e-projetos/25-mercosur-educativo/100-proyecto-caminos-del-mercosur.html

${ }^{8}$ Finalizando el año 2004, se definieron las escuelas que iban a participar del Programa: dos escuelas brasileñas ubicadas en los municipios de Uruguaiana-RS y Dionísio Cerqueira-SC que tienen fronteras con las provincias argentinas de Corrientes y Misiones, respectivamente. Durante 2005, el programa involucró dos escuelas, una en Bernardo de Yrigoyen, Misiones, y la otra en Paso de los Libres, Corrientes. En 2006 el programa se amplió hacia las localidades de Puerto Iguazú, Misiones, y Santo Tomé y La Cruz, Corrientes.

9 Traducción propia.

${ }^{10}$ Traducción propia.

${ }^{11}$ El Informe de gestión del Grupo de Cooperación Descentralizada correspondiente al período 2009/10 se puede ver en: http://www.mercociudades.org/sites/portal.mercociudades.net/files/archivos/documentos/ut/Infor me GCD 2010.pdf

\section{Referencias bibliográficas}

\subsection{Libros, capítulos de libros y artículos especializados}

Ainscow, M., \& Miles, S. (2009). Desarrollando sistemas de educación inclusiva. ¿Cómo podemos hacer progresar las políticas?. En C. Giné (coord.). La educación inclusiva. Barcelona: Horsori, 161-170.

Alburquerque, F. (2001). Desarrollo económico local y cooperación descentralizada para el desarrollo. Desarrollar lo local para una globalización alternativa. San Sebastián, España: HEGOA, Euskal Fondoa.

Arias, A. (2011). Participación Ciudadana y/o canales de participación. Aportes a partir del análisis de las experiencias de "participación" en las áreas de política social asistencial. Revista Demos Participativa.

Axelrod, R., \& Keohane, R. O. (1985). Achieving cooperation under anarchy: Strategies and institutions. World politics, 38(1), 226-254.

Barragán, F. M. (2015). Cooperación transfronteriza: una metodología para su estudio. En Ciencias Sociales y Relaciones Internacionales. Costa Rica: Escuela de Relaciones Internacionales.

Chacón, F. J. S. (2017). Las regiones y su movilización en la integración Andina, Europea y Mercosureña. Referencia a la gobernanza multinivel. Aldea Mundo (36), 23- 36.

Desiderá Neto, W. A., \& Penha, B. (2016). As Regiões de fronteira como laboratório da integração regional no Mercosul. Boletim de Economia e Política Internacional 22.

Durán Jara, D. (2008). El rol del estado en la eficacia y mejora de la educación ¿Hacia una imposición ideológica?. REICE. Revista Iberoamericana sobre Calidad, Eficacia y Cambio en Educación 6, 69-79. Recuperado de: http://www.redalyc.org/articulo.oa?id=55160405

Fagaburu, D. (2017). Afinidades entre la Cooperación Internacional y la Integración Regional en América Latina. Espectros. Revista Cultural 2 (3), 1-39. Recuperado de: http://espectros.com.ar/wp-content/uploads/2017/02/Afinidades-entre-la-CooperaciónInternacional-y-la-Integración-Regional-en-América-Latina_por-Debora-Fagaburu.pdf

Fawcett, L. (2004). Exploring Regional Domains: A Comparative History of Regionalism. International Affairs 80(3), 429-446.

Gomes, J. F. (2017). A contribuição da Rede Mercocidades para o desenvolvimento da integração fronteiriça junto ao Mercosul: o papel da paradiplomacia municipal sul-americana na concretização da integração regional em zonas de fronteiras. Revista Intellector 13(26), 05-19.

Gómez, M., \& Sanahuja, J. A. (1999). El sistema internacional de cooperación al desarrollo. Una aproximación a sus actores e instrumentos. Madrid: CIDEAL.

Hurrell, A. (1995). Teorías de regímenes internacionales: una perspectiva europea. Foro Internacional, octubre-diciembre, 3-31. 
Keohane, R. O. (1993). Institutional theory and the realist challenge after the Cold War. Baldwin, D. (Edit) Neorealism and neoliberalism: The contemporary debate (269-271). EE.UU.: Columbia University Press.

Kooiman, J. (2003). Governing as governance. Sage Publishing.

Malacalza, B. (2013). Estrategia y experimentalismo. Los procesos domésticos de configuración de la Cooperación Sur-Sur desde una perspectiva macro-micro. Un estudio comparado sobre los casos de la Argentina y Brasil (2003-2011). Tesis de doctorado, Facultad Latinoamericana de Ciencias Sociales (FLACSO), Buenos Aires.

Martínez, I., Sanahuja, J. A. (2012). La cooperación descentralizada española y la eficacia de la ayuda: los desafíos de la complementariedad y la división del trabajo. En Martínez, I., Sanahuja, J. A., de la Fuente, R., del Campo, E., \& Verdes-Montenegro, F. J. (Coords.). Eficacia de la ayuda y división del trabajo: Retos para la cooperación descentralizada española (11-27). Recuperado de: http://www.ceipaz.org/images/contenido/COOPERACION\%202012.pdf

Milani, C. R. (2015). International Development Cooperation in the Education Sector: the role of Brazil. EE.UU.: Unesco.

Nye, J. (1990). Soft Power. Foreign Policy, 80.

Oddone, N. (2017). El MERCOSUR post-neoliberal y las fronteras reseteadas: "Si queremos que todo siga como está, necesitamos que todo cambie. En Hernández Ulate A. (et al.). Fronteras latinoamericanas: ejemplos para su comprensión. Costa Rica: Universidad Nacional, Costa Rica. Perrotta, D. (2012). ¿Realidades presentes-conceptos ausentes? La relación entre los niveles nacional y regional en la construcción de políticas de educación superior en el MERCOSUR. Integración y Conocimiento (1).

Posada, E. V. (2005). Evolución de las teorías sobre integración en el contexto de las teorías de relaciones internacionales. Papel político (18), 235-290.

Rhi-Sausi L., J., \& Oddone, N. (2010). Cooperación e integración transfronteriza en el Mercosur: el caso de la Triple Frontera Argentina-Brasil-Paraguay. Maira, L. (ed.). La política internacional subnacional en América Latina (209-258). Buenos Aires: Del Zorzal.

Rhi-Sausi, J. L. (2008). La cooperación internacional en los procesos de descentralización y regionalización de los países latinoamericanos. La experiencia Italia-Región de Atacama.

Rico, A. P. (2017). Políticas de educación inclusiva en América Latina Propuestas, realidades y retos de futuro. Revista de educación inclusiva 3(2).

Riggirozzi, P., \& Tussie, D. (2012). The rise of post-hegemonic regionalism in Latin America. In The Rise of Post-Hegemonic Regionalism (pp. 1-16). Springer Netherlands.

Rose, N. y Miller, P. (1992). Political Power Beyond the State: Problematics of Government. British Journal of Sociology 2 (43).

Sanahuja, J. A. (2014). Enfoques diferenciados y marcos comunes en el regionalismo latinoamericano: Alcance y perspectivas de UNASUR y CELAC. Pensamiento Propio 19, 75-108. Scharpf, F. W. (1996). Negative and Positive Integration in the Political Economy of European Welfare States. En Gary Marks, Fritz W. Scharpf, Philippe C. Schmitter y Wolfgang Streeck (eds.). Governance in the European Union. Londres: Sage.

Schmitter, P. C. (2011). Los conceptos de cooperación e integración regional. Puente@Europa 9(1).

Surasky, J. (2013). La Cooperación Sur-Sur como herramienta decolonial. In Ponencia presentada en el XXVIII Congreso Anual de la Asociación Mexicana de Estudios Internacionales. Huatulco: AMEI.

Torfing, J., \& Díaz-Gibson, J. (2016). Transforming governance to enhance social and educational innovation. Pedagogía Social. Revista Interuniversitaria 28, 99-111.

\subsection{Sitios web consultados}

Mercado Común del Sur (Mercosur). Disponible en: http://www.mercosur.int/innovaportal/v/4054/11/innova.front/textos-fundacionales

Sistema Económico Latinoamericano y del Caribe (SELA). Disponible en: http://sursur.sela.org/qu\%C3\%A9-es-la-css/conceptos-de-lacooperaci\%C3\%B3n-sur-sur/

\subsection{Documentos institucionales e informes técnicos consultados}

Actas del Comité Asesor de Políticas Lingüísticas (CAPL) entre 2006 y 2015.

Actas del Grupo de Cooperación Internacional (GCl) entre 2012 y 2015. 
Actas del Grupo de Trabajo Escuela de Frontera entre 2013 y 2015.

Boletines Oficiales del Mercosur (BOM) entre el 2006 y el 2015.

Decisiones del Consejo del Mercado Común (CMC) entre el 2006 y el 2017.

Decisiones del Grupo Mercado Común entre el 2006 y el 2015.

Organización de las Naciones Unidas para la Educación, la Ciencia y la Cultura (UNESCO), 2011, "Estructura de coordinación de la EPT a nivel global".

Plan Cuatrienal 2006-2010 del Sector Educativo del Mercosur.

Plan Cuatrienal 2011-2015 del Sector Educativo del Mercosur.

Plan Estratégico de Acción Social del Mercosur (PEAS).

Programa Mercosur de la AECID, España. 2011.

SEGIB (2016). La Cooperación Descentralizada en Iberoamérica. Una mirada desde la Cooperación Sur-Sur. Serie - Documentos de trabajo / DT11. Secretaría General Iberoamericana - Programa Iberoamericano para el Fortalecimiento de la Cooperación Sur-Sur.

SEGIB (2017). Lecciones aprendidas y retos para la articulación de actores y la gestión de la Cooperación Descentralizada Sur-Sur. Serie - Documentos de trabajo / DT14. Secretaría General Iberoamericana - Programa Iberoamericano para el Fortalecimiento de la Cooperación Sur-Sur. 